\title{
Acute kidney injury after primary total hip arthroplasty: a risk multiplier for complication, mortality, and healthcare utilization
}

\author{
Jasvinder A. Singh ${ }^{1,2,3^{*}}$ and John D. Cleveland ${ }^{2}$
}

\begin{abstract}
Objective: To assess whether acute kidney injury (AKI) is associated with more complications and higher healthcare utilization in people undergoing primary total hip arthroplasty (THA).

Methods: Using a retrospective cohort study design, we performed multivariable-adjusted logistic regression of the 1998-2014 US National Inpatient Sample data to assess the association of AKI with complications (infection, transfusion, revision, and mortality) and healthcare utilization (total hospital charges, discharge to a rehabilitation facility, length of hospital stay) post-THA. We calculated the odds ratio (OR) and 95\% confidence intervals (CI).

Results: Adjusted for age, gender, race, income, underlying diagnosis, medical comorbidity, and the insurance payer, AKI in people who underwent primary THA was associated with significantly higher $\mathrm{OR}(95 \% \mathrm{Cl})$ of (1) implant infection, 2.34 (95\% Cl, 1.87, 2.93); (2) transfusion, 2.46 (95\% Cl, 2.37, 2.56); (3) revision, 2.54 (95\% Cl, 2.16, 2.98); (4) death, 8.52 (95\% Cl, 7.47, 9.73); (5) total hospital charges above the median, 2.29 (95\% Cl, 1.99, 2.65); (6) discharge to a rehabilitation facility, 2.11 (95\% Cl, 2.02, 2.20); and (7) hospital stay > 3 days, $4.34(95 \%$ Cl, 4.16, 4.53).

Conclusion: Quality improvement initiatives with optimization of the peri-operative care to reduce AKI and subsequently AKI-associated complications and healthcare utilization are needed. Mechanisms of AKI-associated post-THA complications need further examination.
\end{abstract}

Keywords: Acute kidney injury, Total hip arthroplasty, THA, Healthcare utilization, Complications, Outcomes

\section{Background}

Acute kidney injury (AKI) is an uncommon, but a clinically important post-surgical complication. AKI is a risk factor for the development of chronic kidney disease (CKD) and higher mortality [1]. Post-surgical AKI leads to higher adjusted healthcare costs (1.6-times) and longer hospital stays [2]. Thus, post-operative AKI leads to a major healthcare burden.

Total hip arthroplasty (THA) is the second most commonly performed arthroplasty in the US [3] with a growing demand due to associated pain relief and improvement in

\footnotetext{
*Correspondence: Jasvinder.md@gmail.com

${ }^{1}$ Birmingham Veterans Affairs (VA) Medical Center, Birmingham 35233, AL, USA

${ }^{2}$ Department of Medicine at the School of Medicine, University of Alabama at Birmingham, Faculty Office Tower 805B, 510 20th Street S, Birmingham, AL 35294, USA

Full list of author information is available at the end of the article
}

function. The incidence of AKI varied from 1\% [4] to $8 \%$ [5] post-THA in two single-center studies, partially due to differences in the study sample (post-THA versus postTHA or post-TKA), the AKI definition (highest serum creatinine within 5 days post-operative vs. 7 days postoperative), and the time-trends with increased recognition over time (2004-2014 vs. 2013-2014). In a national US sample, the incidence of AKI in a combined sample of people who underwent knee or hip arthroplasty with an underlying diagnosis of osteoarthritis was 1.3\% [6]; no estimates of AKI post-THA or associated complications, such as infection, were available. Complications after THA and knee arthroplasty differ. Most studies of AKI post-THA had significant limitations including single-center studies, small sample sizes, and unadjusted analyses.

(C) The Author(s). 2020 Open Access This article is distributed under the terms of the Creative Commons Attribution 4.0 International License (http://creativecommons.org/licenses/by/4.0/), which permits unrestricted use, distribution, and reproduction in any medium, provided you give appropriate credit to the original author(s) and the source, provide a link to the Creative Commons license, and indicate if changes were made. The Creative Commons Public Domain Dedication waiver (http://creativecommons.org/publicdomain/zero/1.0/) applies to the data made available in this article, unless otherwise stated. 
In the absence of studies of incidence, correlates, and outcomes of AKI post-THA in the US with representative samples, our objective was to examine these aspects of AKI postprimary THA in a representative US sample. We hypothesized a priori that AKI would be associated with a higher rate of complications (infection, revision) and mortality (primary outcomes) after primary THA, and higher healthcare utilization and transfusion rates (secondary outcomes).

\section{Methods}

\section{Data sources and study population}

This retrospective cohort study used the data from the US National (formerly, Nationwide) Inpatient Sample (NIS), a 20\% stratified sample of all discharges from US community hospitals, provided by the Agency for Healthcare Research and Quality (AHRQ) Healthcare Cost and Utilization Project (HCUP) [7]. The University of Alabama at Birmingham's Institutional Review Board (IRB-120207004) approved this study and waived the need for informed consent. Study methods and results are reported in accordance with the Strengthening of Reporting in Observational studies in Epidemiology (STROBE) statement [8] (Additional file 1).

We included all hospitalizations for primary THA in the US from 1998 to 2014, identified by the presence of International Classification of Diseases, ninth revision, common modification (ICD-9-CM) code of 81.51, as the primary procedure code for hospitalization. The codebased algorithm is a validated approach to identify THA cohorts with positive predictive values of 98-99\% [9]. The underlying diagnosis for THA was listed as the primary diagnosis ICD-9-CM code.

\section{Exposure of interest, study outcomes, and covariates}

The exposure of interest, AKI, was identified by the presence of the ICD-9-CM code of 584.5, 584.6, 584.7, 584.8 , or 584.9 as a non-primary (i.e., secondary) diagnosis. This code-based algorithm for AKI is also a validated approach with high positive predictive value of 98\% [10].

Study outcomes were healthcare utilization and inhospital post-operative complications. We assessed the length of hospital stay (above/below the median; 2.7 days rounded off to 3 days), the total hospital charges in US dollars (above/below median for each year), and the discharge disposition, i.e., to home vs. a rehabilitation facility which included short- or long-term care hospital, skilled nursing facility (SNF), intermediate care facility, or a certified nursing facility. Patients who left the hospital against medical advice were considered missing for discharge disposition. The key in-hospital post-operative complications include implant infection, transfusion, and THA revision and mortality.

Important covariates/potential confounders which included demographics (age, sex, race/ethnicity, income), the underlying diagnosis (osteoarthritis (OA), rheumatoid arthritis (RA), fracture, avascular necrosis of bone or other), comorbidity (Deyo-Charlson comorbidity index, a validated measure that included 17 comorbidities, based on the presence of ICD-9-CM codes), insurance payer (Medicare, Medicaid, private insurance, self-pay or other), and hospital characteristics were assessed. Hospital location/teaching status was categorized as rural, urban nonteaching, or urban teaching hospital. Hospital bed size was classified as small, medium, or large, using the NIS cutoffs that vary by the year. Hospital region was categorized as Northeast, Midwest, South, and West.

\section{Statistical analyses}

We performed a separate multivariable-adjusted logistic regression models to assess each clinical (infection, transfusion, THA revision, and mortality) and healthcare utilization outcome (total hospital charges above/below the median, discharge to a rehabilitation facility, length of stay above/below the median of 3 days), adjusting for important potential predictors of post-THA complications and healthcare utilization and/or potential confounders of AKI outcomes [11]. We calculated the odds ratios (OR) and 95\% confidence intervals (CI). We performed sensitivity analyses by additionally adjusting the main analyses for hospital characteristics. We used SAS 9.3 (Cary, NC) for all analyses.

\section{Results}

Of the 4.1 million primary THAs, 61,077 (1.5\%) developed AKI during the index primary THA hospitalization. AKI was associated with higher rate of complications and utilization in unadjusted analyses (Table 1).

Unadjusted estimates were higher for each in-hospital complication (infection, transfusion, revision) by 2 -fold and mortality (20-fold) in people with AKI compared to those without AKI (Table 2). Unadjusted total hospital charges, proportion discharged to a rehabilitation facility, and the length of hospital stay were higher in people with AKI (Table 2).

Adjusted for age, gender, race, income, underlying diagnosis, medical comorbidity, and insurance payer, AKI was associated with significantly higher OR of poorer outcomes: (1) infection, 2.34 (95\% CI, 1.87, 2.93); (2) transfusion, 2.46 (95\% CI, 2.37, 2.56); (3) revision, 2.54 (95\% CI, 2.16, 2.98); (4) death, 8.52 (95\% CI, 7.47, 9.73); (5) total hospital charges above the median, 2.94 (95\% CI, 2.79, 3.03); (6) discharge to a rehabilitation facility, 2.11 (95\% CI, 2.02, 2.20); and (7) the length of hospital stay > 3 days, 4.34 (95\% CI, 4.16, 4.53) (Table 3). Sensitivity analyses that additionally adjusted the main model for hospital characteristics confirmed the main findings with minimal/no attenuation (Table 3). 
Table 1 Demographic and other cohort characteristics for entire primary THA cohort including people without vs. with acute kidney injury (AKI)N (\%), unless specified otherwise; SE standard error

\begin{tabular}{|c|c|c|c|}
\hline & \multirow{2}{*}{$\begin{array}{l}\text { Entire Primary THA Cohort } \\
(N=4,116,485)^{\mathrm{a}}\end{array}$} & \multicolumn{2}{|l|}{ Primary THA } \\
\hline & & No Acute Kidney Injury $(N=4,055,407)^{a}$ & $\overline{\text { Acute Kidney Injury }(N=61,077)^{a}}$ \\
\hline Age, Mean (SE); median & $65.2(0.04) ; 65.9$ & $65.4(0.04) ; 65.9$ & $71.5(0.12) ; 72.2$ \\
\hline \multicolumn{4}{|l|}{ Age category, in years } \\
\hline$<50$ & $449,642(10.9 \%)$ & $446,863(11.0 \%)$ & $2,779(4.5 \%)$ \\
\hline $50-64$ & $1,364,821(33.2 \%)$ & $1,351,472(33.3 \%)$ & $13,349(21.9 \%)$ \\
\hline $65-79$ & $1,732,014(42.1 \%)$ & $1,704,496(42.0 \%)$ & $27,518(45.1 \%)$ \\
\hline$\geq 80$ & $566,521(13.8 \%)$ & $549,094(13.5 \%)$ & $17,427(28.5 \%)$ \\
\hline \multicolumn{4}{|l|}{ Gender } \\
\hline Female & $2,330,188(56.6 \%)$ & $2,301,645(56.8 \%)$ & $28,543(46.7 \%)$ \\
\hline Male & $1,776,722(43.2 \%)$ & $1,744,192(43.0 \%)$ & $32,530(53.3 \%)$ \\
\hline \multicolumn{4}{|l|}{ Race } \\
\hline White & $2,882,041(70.0 \%)$ & $2,838,722(70.0 \%)$ & $43,319(70.9 \%)$ \\
\hline Black & $225,772(5.5 \%)$ & $219,916(5.4 \%)$ & $5,856(9.6 \%)$ \\
\hline Hispanic & $104,385(2.5 \%)$ & $102,556(2.5 \%)$ & $1,829(3.0 \%)$ \\
\hline Other/Missing & $904,234(22.0 \%)$ & $894,166(22.0 \%)$ & $10,068(16.5 \%)$ \\
\hline \multicolumn{4}{|l|}{ Primary Diagnosis } \\
\hline Rheumatoid arthritis (RA) & $29,173(0.7 \%)$ & $29,016(0.7 \%)$ & $157(0.3 \%)$ \\
\hline Avascular bone necrosis & $285,623(6.9 \%)$ & $281,334(6.9 \%)$ & 4,289 (7.0\%) \\
\hline Osteoarthritis (OA) & $3,447,224(83.7 \%)$ & 3,405,538 (84.0\%) & 41,686 (68.3\%) \\
\hline Other & $354,307(8.6 \%)$ & $339,372(8.4 \%)$ & $14,935(24.5 \%)$ \\
\hline Fracture & 117 (0.003\%) & $107(0.003 \%)$ & $10(0.02 \%)$ \\
\hline \multicolumn{4}{|l|}{ Deyo-Charlson Score } \\
\hline 0 & $2,193,575(53.3 \%)$ & $2,181,133(53.8 \%)$ & $12,442(20.4 \%)$ \\
\hline 1 & $926,286(22.5 \%)$ & $913,996(22.5 \%)$ & $12,290(20.1 \%)$ \\
\hline$\geq 2$ & $996,623(24.2 \%)$ & $960,278(23.7 \%)$ & $36,345(59.5 \%)$ \\
\hline \multicolumn{4}{|l|}{ Insurance } \\
\hline Medicaid & $138,809(3.4 \%)$ & $136,558(3.4 \%)$ & $2,251(3.7 \%)$ \\
\hline Medicare & $2,234,675(54.3 \%)$ & $2,190,406(54.0 \%)$ & $44,269(72.5 \%)$ \\
\hline Other & $102,277(2.5 \%)$ & 101,017 (2.5\%) & $1,260(2.1 \%)$ \\
\hline Private & $1,600,829(38.9 \%)$ & $1,588,027(39.2 \%)$ & $12,802(21.0 \%)$ \\
\hline Self & $32,307(0.8 \%)$ & $31,939(0.8 \%)$ & $368(0.6 \%)$ \\
\hline \multicolumn{4}{|l|}{ Income Category ${ }^{\mathrm{b}}$} \\
\hline $0-25^{\text {th }}$ percentile & $653,243(15.9 \%)$ & $639,522(15.8 \%)$ & $13,721(22.5 \%)$ \\
\hline $25-50^{\text {th }}$ percentile & $1,009,678(24.5 \%)$ & $994,108(24.5 \%)$ & $15,570(25.5 \%)$ \\
\hline $50-75^{\text {th }}$ percentile & $1,086,953(26.4 \%)$ & $1,071,309(26.4 \%)$ & $15,644(25.6 \%)$ \\
\hline $75-100^{\text {th }}$ percentile & $1,285,854(31.2 \%)$ & $1,270,801(31.3 \%)$ & $15,053(24.6 \%)$ \\
\hline \multicolumn{4}{|l|}{ Hospital Location/Teaching } \\
\hline Rural & $444,188(10.8 \%)$ & $439,410(10.8 \%)$ & $4,778(7.8 \%)$ \\
\hline Urban & $1,722,390(41.8 \%)$ & $1,696,986(41.8 \%)$ & $25,404(41.6 \%)$ \\
\hline Urban Teaching & $1,939,988(47.1 \%)$ & $1,909,531(47.1 \%)$ & 30,457 (49.9\%) \\
\hline \multicolumn{4}{|l|}{ Hospital Bed size } \\
\hline Small & $685,208(16.6 \%)$ & 676,555 (16.7\%) & 8,653 (14.2\%) \\
\hline Medium & $1,037,562(25.2 \%)$ & $1,021,335(25.2 \%)$ & $16,227(26.6 \%)$ \\
\hline
\end{tabular}


Table 1 Demographic and other cohort characteristics for entire primary THA cohort including people without vs. with acute kidney injury (AKI) (Continued)

\begin{tabular}{|c|c|c|c|}
\hline & \multirow{2}{*}{$\begin{array}{l}\text { Entire Primary THA Cohort } \\
(N=4,116,485)^{\mathrm{a}}\end{array}$} & \multicolumn{2}{|l|}{ Primary THA } \\
\hline & & No Acute Kidney Injury $(N=4,055,407)^{a}$ & Acute Kidney Injury $(N=61,077)^{c}$ \\
\hline Large & $2,383,797(57.9 \%)$ & $2,348,038(57.9 \%)$ & $35,759(58.5 \%)$ \\
\hline \multicolumn{4}{|c|}{ Hospital Region } \\
\hline Northeast & $818,699(19.9 \%)$ & $807,610(19.9 \%)$ & $11,089(18.2 \%)$ \\
\hline Midwest & $1,089,883(26.5 \%)$ & $1,073,918(26.5 \%)$ & $15,965(26.1 \%)$ \\
\hline South & $1,358,857(33.0 \%)$ & $1,334,688(32.9 \%)$ & 24,169 (39.6\%) \\
\hline West & $849,046(20.6 \%)$ & $839,192(20.7 \%)$ & $9,854(16.1 \%)$ \\
\hline
\end{tabular}

${ }^{a}$ The national estimates were based on the following from the $20 \%$ NIS sample: Entire cohort $(N=855,634) ;$ no AKI $(N=843,044) ; A K I(N=12,590)$

bIncome quartiles were defined as follows: (1) from 2003, ZIPINC_QRTL variable is available, which was used; (2) 1998-2002: another categorical variable (ZIPINC) indicated the median household income of the patient's residential ZIP Code, based on the 1999 demographics. The categories were defined so that the maximum for category $1(\$ 25,000)$ was approximately $150 \%$ of the 1999 poverty level and the boundary between the second and third categories $(\$ 35,000)$ was approximately the national median household income. As an example, for $2014, \mathrm{Q} 1\left(0-25^{\text {th }}\right.$ percentile) was $\$ 1-39,999, \mathrm{Q} 2$ was $\$ 40,000-50,999, \mathrm{Q} 3$ was $\$ 51,000-$ 65,999 ; and Q4 was $\$ 66,000+$. In 1998 , Q1 was $\$ 1-28,999$, Q2 $\left(25-50^{\text {th }}\right.$ percentile) was $\$ 29,000-36,999$, Q3 (50-75 ${ }^{\text {th }}$ percentile) was $\$ 37,000-49,999 ;$ and Q4 (75$100^{\text {th }}$ percentile) was $\$ 50,000+$

Table 2 Unadjusted estimates for in-hospital complications and healthcare utilization outcomes for the index hospitalization for primary THA for the entire cohort and by the presence/absence of AKI

\begin{tabular}{|c|c|c|c|}
\hline & \multirow{2}{*}{$\begin{array}{l}\text { Entire Primary THA } \\
\text { Cohort }(N=4,116, \\
485)^{\mathrm{a}}\end{array}$} & \multicolumn{2}{|l|}{ Primary THA } \\
\hline & & $\begin{array}{l}\text { No Acute Kidney Injury }(N=4,055 \text {, } \\
407)^{\mathrm{a}}\end{array}$ & $\begin{array}{l}\text { Acute Kidney Injury }(N=61,077) \\
\text { a }\end{array}$ \\
\hline \multicolumn{4}{|l|}{ In-hospital Complications ${ }^{\mathrm{b}}$} \\
\hline Infection & $7,592(0.2 \%)$ & $7,173(0.2 \%)$ & $419(0.7 \%)$ \\
\hline Revision & $17,931(0.4 \%)$ & $17,252(0.4 \%)$ & $679(1.1 \%)$ \\
\hline Transfusion & $937,803(22.8 \%)$ & $912,311(22.5 \%)$ & $25,492(41.7 \%)$ \\
\hline Death & $8,889(0.2 \%)$ & $6,538(0.2 \%)$ & $2,351(3.8 \%)$ \\
\hline \multicolumn{4}{|l|}{ Healthcare Utilization Outcomes } \\
\hline \multicolumn{4}{|l|}{ Discharge Status } \\
\hline Home & $2,448,107(59.5 \%)$ & $2,427,284(59.9 \%)$ & $20,823(34.1 \%)$ \\
\hline Inpatient facility ${ }^{c}$ & $1,649,102(40.1 \%)$ & $1,611,362(39.7 \%)$ & $37,740(61.8 \%)$ \\
\hline Length of hospital stay, Mean (SE); median & $3.71(0.01) ; 2.74$ & $3.68(0.01) ; 2.74$ & $7.15(0.07) ; 4.49$ \\
\hline \multicolumn{4}{|l|}{ Length of hospital stay categories } \\
\hline$\leq 3$ days & $2,499,883(60.7 \%)$ & $2,483,373(61.2 \%)$ & $16,510(27.0 \%)$ \\
\hline$>3$ days & $1,616,602(39.3 \%)$ & $1,572,034(38.8 \%)$ & $44,568(73.0 \%)$ \\
\hline $\begin{array}{l}\text { Total Hospital Charges, in U.S. \$, Mean (SE); } \\
\text { Median }\end{array}$ & $44,635(268) ; 37,658$ & $44,313(266) ; 37,537$ & $81,202(911) ; 61,686$ \\
\hline $1998-2000$ & $23,556(275) ; 20,858$ & $23,455(274) ; 20,838$ & $56,412(3,039) ; 39,461$ \\
\hline $2001-2002$ & $29,210(431) ; 25,432$ & $29,044(427) ; 25,398$ & $63,621(3,521) ; 41,480$ \\
\hline 2003-2004 & $36,086(598) ; 31,359$ & $35,805(597) ; 31,289$ & $72,805(3,355) ; 49,318$ \\
\hline $2005-2006$ & $40,678(623) ; 35,383$ & $40,369(621) ; 35,290$ & 70,439 (2,308); 50,555 \\
\hline $2007-2008$ & $47,216(787) ; 41,423$ & 46,764 (777); 41,291 & 77,075 (2,668); 55,301 \\
\hline $2009-2010$ & $\begin{array}{l}49,918(1,062) ; 43 \\
304\end{array}$ & $49,305(1,054) ; 43,022$ & $77,483(1,894) ; 60,193$ \\
\hline $2011-2012$ & 56,395 (848); 48,917 & $55,681(833) ; 48,600$ & $86,252(2,211) ; 67,974$ \\
\hline 2013-2014 & 58,964 (532); 51,157 & 58,230 (528); 50,851 & $90,271(1,775) ; 69,643$ \\
\hline
\end{tabular}

$\mathrm{N}(\%)$, unless specified otherwise; SE standard error

${ }^{a}$ The national estimates were based on the following from the $20 \%$ NIS sample: Entire cohort $(N=855,634)$; no AKI $(N=843,044) ; A K I(N=12,590)$

b In-hospital complications during the index hospitalization for primary THA

${ }^{c}$ Inpatient facility included short- or long-term care hospital, skilled nursing facility (SNF), intermediate care facility, or a certified nursing facility 
Table 3 Multivariable-adjusted association of AKI with healthcare utilization outcomes and in-hospital complications for index hospitalization for primary THA in the main model and sensitivity analysis

\begin{tabular}{|c|c|c|}
\hline & Main Model ${ }^{a}$ & $\begin{array}{l}\text { Sensitivity analyses }{ }^{\mathrm{b}} \text { : } \\
\text { Main Model + hospital characteristics }\end{array}$ \\
\hline & OR $(95 \% \mathrm{Cl})$ & OR $(95 \% \mathrm{Cl})$ \\
\hline Total hospital charge above the median ${ }^{c}$ & $2.29(1.99,2.65)$ & $2.25(1.95,2.60)$ \\
\hline Discharge to an inpatient/rehabilitation facility ${ }^{d}$ & $2.11(2.02,2.20)$ & $2.15(2.06,2.25)$ \\
\hline Length of hospital stay more than median $\left(>3\right.$ days) ${ }^{e}$ & $4.34(4.16,4.53)$ & $4.39(4.20,4.58)$ \\
\hline \multicolumn{3}{|l|}{ In-hospital complications ${ }^{f}$} \\
\hline Infection & $2.34(1.87,2.93)$ & $2.33(1.87,2.91)$ \\
\hline Transfusion & $2.46(2.37,2.56)$ & $2.52(2.42,2.61)$ \\
\hline Revision & $2.54(2.16,2.98)$ & $2.56(2.17,3.01)$ \\
\hline Death & $8.52(7.47,9.73)$ & $8.54(7.48,9.76)$ \\
\hline
\end{tabular}

BOLD indicates significant odds ratio

${ }^{a}$ Main model was adjusted for socio-demographics (age, race/ethnicity, gender, income), the Deyo-Charlson comorbidity index, insurance payer and the underlying diagnosis for primary THA

${ }^{b}$ Sensitivity analyses adjusted each main model additionally for hospital characteristics including hospital location/teaching status, hospital region and hospital bed size

${ }^{\mathrm{C}}$ Median hospital charges were calculated for each year

dInpatient/rehabilitation facility included short- or long-term care hospital, skilled nursing facility (SNF), intermediate care facility, or a certified nursing facility ${ }^{\mathrm{e}}$ Median length of hospital stay was 2.74 days, rounded off to 3 days

f In-hospital complications

\section{Discussion}

In this national study of $>4$ million primary THAs using the 1998-2014 US NIS, we found the diagnosis of AKI in $1.5 \%$ of all primary THA hospitalizations, similar to the incidence of $1.3-1.5 \%$ reported previously in a combined knee/hip cohort or from a single-center $[4,6]$. To our knowledge, this is the first study to provide data on AKI post-THA based on the redesign of NIS in 2012 [7] intended to improve the national estimates. Several findings merit further discussions.

The absolute increase in post-primary-THA complications with AKI ranged 0.5\% for implant infection to 19\% for transfusion. This finding is clinically important, given that the rate of complications and mortality rates in those without AKI were $0.2-0.4 \%$ except that for transfusion (22.5\%). The multivariable-adjusted relative risk of in-hospital complications was 2.3-2.5 times. Most primary THAs are elective procedures. Our findings identify AKI as an important risk factor for complications post-primary THA.

Potential mechanisms of post-operative AKI involve hemodynamic, inflammatory, and nephrotoxic factors [12], including angiotensin-converting enzyme inhibitor/ angiotensin receptor blockers (ACE inhibitors/ARBs) and non-steroidal anti-inflammatory drugs (NSAIDs), which can be associated with added toxicity when used concomitantly with each other. American Society of Anesthesiologist (ASA) score, cardiovascular disease, diabetes, creatinine $>2 \mathrm{mg} / \mathrm{dl}$, and high-risk surgery were significant predictors of post-operative AKI [13]. In THA populations, NSAIDs were either associated with 2.7-times higher risk of AKI [14] or not associated with
AKI post-THA $[4,5]$. A recent review identified obesity, metabolic syndrome, perioperative antibiotics, antihypertensives medications, and antibiotic-impregnated cement spacers as risk factors for AKI post-knee arthroplasty [15]. These findings may likely applicable to post-THA AKI. Patient education, careful avoidance of nephrotoxic medications in peri-operative including those leading to hypotension, maintenance of adequate intravascular volume, and effective comorbidity management (cardiac, vascular, pulmonary, renal, diabetes, etc.) may also help in reducing the risk of post-THA AKI. Studies of quality initiatives of these interventions (alone or in combination) are needed to generate evidence to support these approaches in clinical practice.

AKI during index primary THA hospitalization was independently associated with higher healthcare utilization in multivariable-adjusted analyses. Our study found a 3.5 -day longer hospital stay and $\$ 37,000$ excess mean hospital charges (unadjusted) and 8.5-fold higher adjusted mortality associated with AKI post-THA, extends findings from a single-center study [16] and from a study that combined primary and revision knee/hip arthroplasty performed only for osteoarthritis [6]. AKI was also associated with a longer hospital stay and 2.1times higher odds of discharge to a rehabilitation facility.

Study strengths include the use of a national, representative sample and adjustment for several potential confounders. Our study findings must be interpreted carefully considering the study limitations. The NIS excludes federal facilities such as military hospitals and Veterans Affairs medical centers, and therefore, the findings are not generalizable to these cohorts. The NIS 
counts hospitalizations and not procedures, i.e., bilateral procedures are missed and counted as single procedures. Since simultaneous bilateral THAs are uncommon $(<$ $1 \%$ ), this is unlikely to have affected our estimates greatly. We examined hospital charges, not actual hospital costs or what was reimbursed, which is usually lower than the charges. Misclassification bias is possible, since we used ICD-9-CM codes to identify both AKI and TKA, despite a high validity of codes for both conditions $[9,10]$. Longitudinal follow-up is desirable for post-THA outcomes; however, NIS data do not lend themselves to such analyses. Another limitation is the lack of a published protocol for this observational study, although we specified more complications, and higher healthcare utilization and mortality rate associated with AKI post-THA a priori.

\section{Conclusions}

In conclusion, in a national cohort of patients undergoing primary THA, AKI occurred in $1.5 \%$ of all hospitalizations. AKI in patients who underwent primary THA was independently associated with each of the four postoperative in-hospital complications in adjusted analyses, including mortality. AKI was also associated with higher healthcare utilization post-THA in adjusted analyses. Quality initiatives that further improve the care pathways for patients undergoing primary THA may reduce the risk of AKI, and associated complications and excess healthcare utilization. Research studies need to explore the mechanisms of AKI in patients undergoing primary THA and examine interventions that target modifiable risk factors for AKI.

\section{Supplementary information}

Supplementary information accompanies this paper at https://doi.org/10. $1186 /$ s13075-020-2116-3.

Additional file 1. Mapping of the various components in our manuscript of the STROBE Statement checklist of items for reports of observational studies.

\section{Abbreviations}

AKI: Acute kidney injury; THA: Total hip arthroplasty; NIS: National (formerly, Nationwide) Inpatient Sample; AHRQ: Agency for Healthcare Research and Quality; HCUP: Healthcare Cost and Utilization Project; ICD-9-

CM: International Classification of Diseases, ninth revision, common modification; US: United States; OR: Odds ratio; Cl: Confidence intervals; SE: Standard error of the mean

\section{Acknowledgements}

None

\section{Authors' contributions}

JAS designed the study, developed the study protocol, reviewed the analyses, wrote the first draft of the paper, and made the decision to submit the final manuscript. JDC performed the data analyses, reviewed the analyses, and revised the manuscript. JDC had full access to all of the data in the study and takes the responsibility for the integrity of the data and accuracy of the data analysis. The author(s) read andapproved the final manuscript.

\section{Funding}

This material is the result of work supported by research funds from the Division of Rheumatology at the University of Alabama at Birmingham and the resources and use of facilities at the Birmingham VA Medical Center, Alabama, USA, to the study PI (JAS). No grant funding was obtained for this study.

\section{Availability of data and materials}

We are ready to share the data with colleagues, after obtaining appropriate permissions from the University of Alabama at Birmingham (UAB) ethics board and done in compliance the UAB patient privacy rules and data sharing policies.

\section{Ethics approval and consent to participate}

The study was approved by the Institutional Review Board (IRB) at the University of Alabama at Birmingham (UAB; IRB-120207004).

\section{Consent for publication}

No individual person's data were presented in any form in this study, and therefore, no consent to publish is required.

\section{Competing interests}

There are no financial conflicts related directly to this study. JAS has received consultant fees from Crealta/Horizon, Medisys, Fidia, UBM LLC, Medscape, WebMD, Clinical Care options, Clearview healthcare partners, Putnam associates, Spherix, the National Institutes of Health, and the American College of Rheumatology. JAS owns stock options in Amarin pharmaceuticals and Viking therapeutics. JAS is a member of the executive of OMERACT, an organization that develops outcome measures in rheumatology and receives arms-length funding from 36 companies. JAS is a member of the Veterans Affairs Rheumatology Field Advisory Committee. JAS is the editor and the Director of the UAB Cochrane Musculoskeletal Group Satellite Center on Network Meta-analysis. JAS previously served as a member of the following committees: member, the American College of Rheumatology's (ACR) Annual Meeting Planning Committee (AMPC) and Quality of Care Committees; the Chair of the ACR Meet-the-Professor, Work shop and Study Group Subcommittee; and the co-chair of the ACR Criteria and Response Criteria subcommittee. The other author declares that there are no competing interests.

\section{Author details}

'Birmingham Veterans Affairs (VA) Medical Center, Birmingham 35233, AL, USA. ${ }^{2}$ Department of Medicine at the School of Medicine, University of Alabama at Birmingham, Faculty Office Tower 805B, 510 20th Street S, Birmingham, AL 35294, USA. ${ }^{3}$ Division of Epidemiology at the School of Public Health, University of Alabama at Birmingham, Faculty Office Tower 805B, 510 20th Street S, Birmingham, AL 35294, USA.

Received: 20 September 2019 Accepted: 4 February 2020

Published online: 19 February 2020

\section{References}

1. 1990 Census. We asked.......You told us. Race. http://www.census.gov// apsd/cqc/cqc4.pdf. In. Edited by Commerce USDo. Washington, D.C.: U.S. Census Bureau; 1992.

2. Hobson C, Ozrazgat-Baslanti T, Kuxhausen A, Thottakkara P, Efron PA, Moore FA, Moldawer LL, Segal MS, Bihorac A. Cost and mortality associated with postoperative acute kidney injury. Ann Surg. 2015;261(6):1207-14.

3. Singh JA, Vessely MB, Harmsen WS, Schleck CD, Melton $L$ 3rd, Kurland RL, Berry DJ. A population-based study of trends in the use of total hip and total knee arthroplasty, 1969-2008. Mayo Clin Proc. 2010;85(10):898-904.

4. Gharaibeh KA, Hamadah AM, Sierra RJ, Leung N, Kremers WK, El-Zoghby $\mathrm{ZM}$. The rate of acute kidney injury after total hip arthroplasty is low but increases significantly in patients with specific comorbidities. J Bone Joint Surg Am. 2017;99(21):1819-26.

5. Ferguson KB, Winter A, Russo L, Khan A, Hair M, MacGregor MS, Holt G. Acute kidney injury following primary hip and knee arthroplasty surgery. Ann R Coll Surg Engl. 2017;99(4):307-12.

6. Nadkarni GN, Patel AA, Ahuja Y, Annapureddy N, Agarwal SK, Simoes PK, Konstantinidis I, Kamat S, Archdeacon M, Thakar CV. Incidence, risk factors, 
and outcome trends of acute kidney injury in elective total hip and knee arthroplasty. Am J Orthop (Belle Mead NJ). 2016;45(1):E12-9.

7. HCUP Databases. Healthcare Cost and Utilization Project (HCUP). Overview of the Nationwide Inpatient Sample (NIS). http://www.hcup-us.ahrq.gov/ nisoverview.jsp. Last modified 8/13/18.

8. STROBE Statement. Strengthening the reporting of observational studies in epidemiology. Link: http://www.strobe-statement.org/fileadmin/Strobe/ uploads/checklists/STROBE_checklist_v4_cohort.pdf Accessed 02/28/2017.

9. Katz JN, Losina E, Barrett J, Phillips CB, Mahomed NN, Lew RA, Guadagnoli E, Harris WH, Poss R, Baron JA. Association between hospital and surgeon procedure volume and outcomes of total hip replacement in the United States Medicare population. J Bone Joint Surg Am. 2001;83-A(11):1622-9.

10. Waikar SS, Wald R, Chertow GM, Curhan GC, Winkelmayer WC, Liangos O, Sosa MA, Jaber BL. Validity of international classification of diseases, ninth revision, clinical modification codes for acute renal failure. J Am Soc Nephrol. 2006;17(6):1688-94.

11. Santaguida PL, Hawker GA, Hudak PL, Glazier R, Mahomed NN, Kreder HJ, Coyte PC, Wright JG. Patient characteristics affecting the prognosis of total hip and knee joint arthroplasty: a systematic review. Can J Surg. 2008;51(6): 428-36.

12. Rosner MH, Okusa MD. Acute kidney injury associated with cardiac surgery. Clin J Am Soc Nephrol. 2006;1 (1):19-32.

13. Abelha FJ, Botelho M, Fernandes V, Barros H. Determinants of postoperative acute kidney injury. Crit Care. 2009:13(3):R79.

14. Warth LC, Noiseux NO, Hogue MH, Klaassen AL, Liu SS, Callaghan JJ. Risk of acute kidney injury after primary and revision total hip arthroplasty and total knee arthroplasty using a multimodal approach to perioperative pain control including ketorolac and celecoxib. J Arthroplast. 2016;31(1):253-5.

15. Siddiqi A, White PB, Etcheson JI, George NE, Gwam CU, Mistry JB, Patel NG, Adam H, Delanois RE. Acute kidney injury after total knee arthroplasty: a clinical review. Surg Technol Int. 2017;31:243-52.

16. Chertow GM, Burdick E, Honour M, Bonventre JV, Bates DW. Acute kidney injury, mortality, length of stay, and costs in hospitalized patients. J Am Soc Nephrol. 2005;16(11):3365-70

\section{Publisher's Note}

Springer Nature remains neutral with regard to jurisdictional claims in published maps and institutional affiliations.

Ready to submit your research? Choose BMC and benefit from:

- fast, convenient online submission

- thorough peer review by experienced researchers in your field

- rapid publication on acceptance

- support for research data, including large and complex data types

- gold Open Access which fosters wider collaboration and increased citations

- maximum visibility for your research: over $100 \mathrm{M}$ website views per year

At $\mathrm{BMC}$, research is always in progress.

Learn more biomedcentral.com/submissions 